\title{
Die Algenvegetation von List auf Sylt
}

\author{
Von Peter Kornmann \\ Aus der Biologischen Anstalt Helgoland in List auf Sylt, \\ Forschungsinstitut der Bundesanstalt für Fischerei \\ (Mit 1 Abbildung im Text)
}

Die Algenflora in dem Gebiet um List auf Sylt steht zwar an Artenzahl und Besiedelungsdichte weit hinter der Helgolands zurück, nimmt aber dennoch eine bevorzugte Stellung in der Deutschen Bucht ein. Die Liste der hier gefundenen Arten ist größer, als nach den wenigen Angaben in der Literatur zu erwarten war. Reinbold (1892) bezeichnet Sylt im Vergleich zu Amrum und Föhr als artenarm. In Rosenvinge's (1935) Zusammenstellung über die Verteilung der Rotalgen in den dänischen Gewässern werden für das nördlich benachbarte Gebiet der Inseln Röm und Fanö nur 10 Rotalgen angegeben, wobei noch hervorgehoben wird, daß dieser Bezirk günstigere Bedingungen für die Algenansiedlung bietet als andere Stellen des südlichen Teils der jütländischen Küste. Demgegenüber wurden bei List bisher 65 Arten aufgefunden, darunter 20 Rotalgen.

Als Grundlage für die folgende Darstellung dienten mir die durch die Verlegung der Biologischen Anstalt Helgoland nach List auf Sylt seit dem Jahre 1946 von dem technischen Assistenten Herrn P. H. SAHLING gesammelten Algen sowie meine eigenen Beobachtungen seit Juni 1950. Das Laboratorium der Biologischen Anstalt liegt nahezu am Ostende des Ellenbogens, einer schmalen Halbinsel, in die die Insel Sylt an ihrem nördlichen Ende nach Osten umbiegt (siehe $\mathrm{Abb}$.). Diese fast ganz von Dünen eingenommene Landzunge grenzt im Norden an das Lister Tief, das den Wasseraustausch der Watten zwischen dem Sylter und Römer Festlandsdamm im Wechsel der Gezeiten vermittelt. Im Süden dehnt sich der Königshafen aus, eine Bucht des Wattenmeeres. Beide Küsten sind sandig, jedoch ist an einzelnen Stellen verhältnismäßig festliegendes Geröll abgelagert und damit die Vorausseţung für die Ansiedlung von Algen gegeben. Die unterschiedlichen Standortbedingungen auf der See- und Wattenmeerseite steigern naturgemäß die Mannigfaltigkeit der Algenvegetation.

Günstige Bedingungen für den Algenanwuchs bieten die in dem Gebiet vorhandenen künstlichen Bauten: die Buhnen am Nordstrand, die Anlegebrücke bei der Station sowie Reste ehemaliger Löschbrücken, eine große Buhne im Süden von List, die Brückenpfähle im Lister Hafen, eine auf die Reede auslaufende Ablaufbahn sowie Uferbefestigungen nördlich des Hafens. Auch die Miesmuschelbänke im Königshafen sind dicht von Algen bewachsen. Im übrigen aber ist der Untergrund im Gebiet um List beweglich und großenteils stärkeren 
Verlagerungen durch das strömende Wasser ausgesetgt. Daher werden geschlossene Algenformationen wie auf dem Helgoländer Felswatt im allgemeinen nicht angetroffen.

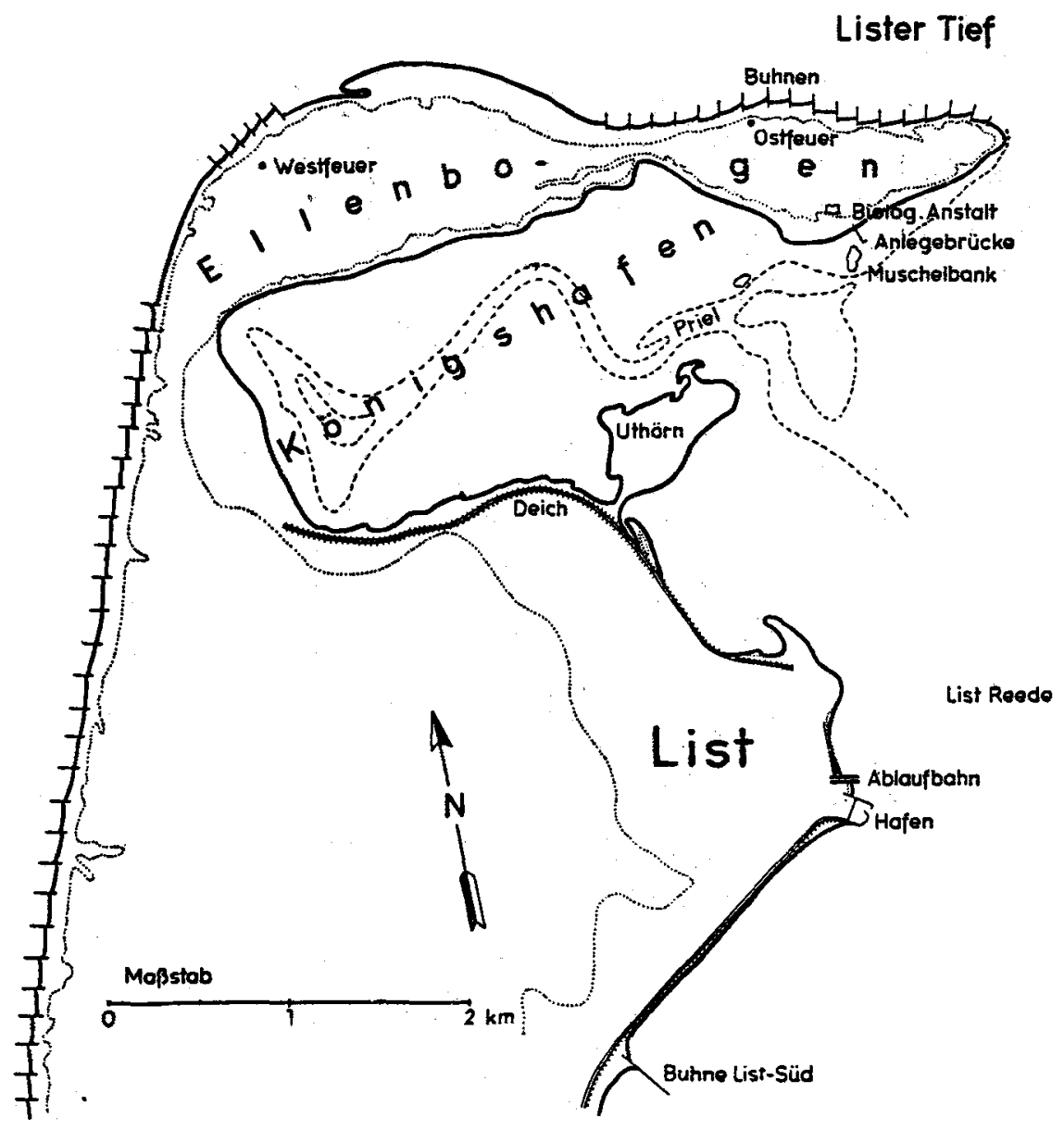

Von den hydrographischen Bedingungen stände der Salzgehalt des Meerwassers mit Werten zwischen 30 und $32 \%$ der Entfaltung einer reichhaltigeren Algenflora nicht entgegen. Auch die sommerlichen Temperaturen, die ausnahmsweise einmal $21^{\circ} \mathrm{C}$ erreichen können, stellen keinen begrenzenden Faktor dar. Dagegen könnten die tiefen Temperaturen im Winter bis zu $-1,6^{\circ} \mathrm{C}$ - tägliche Temperaturmessungen werden bei Hochwasser an der Anlegebrücke seit April 1948 durchgeführt - das Vorkommen mancher Arten ausschließen. Während solcher Kälteperioden fallen weite Gebiete des Wätts trocken und sind den niedrigen Lufttemperaturen ausgesetst. Dazu kommt eine starke Eisbildung; die durch die Gezeitenströmung bewegten Eismassen können stellenweise rein mechanisch den Algenbewuchs vernichten.

Durch die örtlichen Gegebenheiten bedingt, gehören die meisten der bei List gefundenen Algen der litoralen Zone an, dem Gezeitengürtel zwischen der mittleren Tiden-Hoch- und Tiden-Niedrigwasserlinie, einige Arten bevorzugen die Zone dicht unter der Tiden-Niedrigwassergrenze. Zur Ausbildung. eines supralitoralen Bereichs kommt es bei dem überall flachen Strande nicht, 
wenn man nicht die an einzelnen Stellen anzutreffende Vaucheria- und Cyanophyceen-Formation dazu rechnen will. Auch in der sublitoralen Zone fehlt im allgemeinen der geeignete Untergrund für die Ansiedelung ihrer typischen Vertreter. Die Vegetation auf den Austernbänken in 3-4 m Tiefe weist nach den bisher untersuchten Dretschproben keine Arten auf, die in der Gezeitenzone fehlen.

Die Küste längs der Westseite der Insel Sylt und der Nordseite des Ellenbogens ist ein flacher Sandstrand. Schon in geringer Entfernung landeinwärts erheben sich die Dünen; bei Sturmfluten sind sie dem Angriff der Brandung ausgesetgt. Durch Buhnenbauten wirkt man dem fortschreitenden Landverlust und einer Rückverlegung der Küste entgegen. Eiserne Spundbohlenwände im Abstand von $100 \mathrm{~m}$ unterteilen auch die Nordküste des Ellenbogens in einzelne Kammern. In diesen finden sich stellenweise Geröllablagerungen in einem schmalen Streifen um die Tiden-Niedrigwassergrenze, auf denen sich im Sommer ein dichter Algenteppich entwickelt. Hier siedeln sich Enteromorpha compressa (L.) Grev., E. lingulata J. G. Ag. und E. linza (L.) J. G. Ag., Ulva lactuca L. und mehrere Cladophora-Arten an; Ceramium rubrum (Huds.) Ag., Polysiphonia nigrescens (Engl. Bot.) Grev. und zeitweilig auch P. atrorubescens (Dillw.) Grev. kommen reichlich vor. Dazwischen findet sich Scytosiphon lomentaria (Lyngb.) J. G. Ag., oftmals mit mancherlei Epiphyten besetst. Chorda filum (L.) Stadkh. wächst dicht unter der Niedrigwassergrenze, so daß ihre Fäden bei tiefem Wasserstand wie eine Decke an der Wasseroberfläche fluten. Auf Steinen und als Epiphyten sowie an den Buhnenköpfen finden sich Ectocarpus-Arten ein: E. siliculosus (Dillw.) Lyngb., E. confervoides (Roth) Le Jol. und E. granulosus (Engl. Bot.) Ag. werden angetroffen.

Die Spundwände selbst sind nur auf ihrer Ostseite bis an die Strandlinie dicht mit Algen bewachsen. Auf der Westseite beginnt der Bewuchs erst weiter draußen, weil die Brandungswogen vielfach Sand und kleine Steine mitführen und dadurch den jungen Anwuchs abscheuern. Lediglich kleine Fadenalgen wie Bangia fuscopurpurea (Dillw.) Lyngb. und Urospora penicilliformis (Roth) Aresch. hatten sich im Dezember 1950 nach einer längeren, für eine ungestörte Entwicklung günstigen Wetterperiode entwickelt. Die Ostseite der Spundwände trägt eine geschlossene Besiedlung von Balanus balanoides, der von der NWLinie bis etwa $1 \mathrm{~m}$ unter die Tiden-HW-Linie reicht. Die Balanus-Zone ist nahezu in ihrer ganzen Höhe mit Fucus platycarpus Thur. bedeckt. Fucus vesiculosus $\mathrm{L}$, den man in ihrer unteren Hälfte erwarten sollte, fehlt. Uber dem Fucus zieht sich ein Band von Enteromorpha minima Küts. hin, darüber siedeln sich fädige Algen wie Bangia fuscopurpurea (Dillw.) Lyngb., Ulothrix implexa Kütz. und U. flacca (Dillw.) Thur. sowie Urospora penicilliformis (Roth) Aresch. an. Porphyra umbilicalis (L.) Küts. fehlt nicht, sie streut sich in den Fucus- und den Enteromorpha minima-Bestand ein. Auf einer alten Steinbuhne in der Nähe des Lister Westfeuers fanden sich an einer Stelle, die stärkerem Wellenschlag ausgesett ist, Nemalion multifidum. (Web. et Mohr) J. Ag. und Polysiphonia urceolata (Dillw.) Grev. gerade an der Niedrigwassergrenze. Beide Algen wurden auch an Buhnen und Findlingsblöcken vor dem Roten Kliff bei Kampen festgestellt.

Diese ergiebigen Algenplätze am Nordstrand sind leider gelegentlichen $V$ eränderungen unterworfen. Bei anhaltend ablandigem Wind kann der in unmittelbarer Nähe am Vorstrand liegende Sand in solchen Mengen verweht werden, daß er die nur schmalen Geröllstellen und Tẹile der Buhnen über- 
schüttet. Wenn dann nach einiger Zeit Brandung und Strömung den Sand wieder weggeführt haben, so wird zwar das Geröll schnell wieder von rasch wachsenden Algen besiedelt, die Fucus-Vegetation an den Spundwänden aber kann sich erst nach längerer Zeit ungestörter Entwicklung wieder einstellen.

Ganz andere und recht eigenartige Vegetationsverhältnisse bieten sich auf dem Watt $z$ wischen der Ellenbogen-Halbinsel und dem Königshafen-Priel. Auf den großen, bei Niedrigwasser trocken fallenden, schwach geneigten Sandflächen findet man in ganz lockerer Verteilung einzelne Algenbüschel. Sie sind an Steinen, lebenden Herz- oder Miesmuscheln oder auch an Muschelschalen angeheftet. Vielfach besteht der Anker aus Schalenresten und Steinen, die durch Byssusfäden miteinander verklumpt sind. Auf diesem "Substrat" können mehrere Algenarten zugleich Fuß gefaßt haben. Oftmals steckt der Anker mehrere Zentimeter tief im Sand. Nach stärkerem Wellengang findet man die Algenbüschel samt ihrer Unterlage zahlreich im Spülsaum ausgeworfen, die Algen werden also samt ihrem Substrat leicht verfrachtet. Es kommen im allgemeinen 1-3 Algenbüschel auf $1 \mathrm{~m}^{2}$, stellenweise mehr oder weniger. Folgende Arten trifft man in der geschilderten Weise auf dem Watt wachsend an: Gracilaria confervoides (L.) Grev., Dumontia incrassata (Müll.) Lamour., F'olysiphonia nigrescens (Engl. Bot.) Grev., P.elongata (Huds.) Harv. und P. atrorubescens (Dillw.) Grev., Ceramium rubrum (Huds.) Ag., Scytosiphon lomentaria (Lyngb.) J. G. Ag., Chaetomorpha aerea (Dillw.) Kütz. und Enteromorpha compressa (L.) Grev. Im Juli bis September ist Gracilaria reichlich mit dem zierlichen Callithamnion corymbosum (Eng. Bot.) Lyngb. besett.

An zwei Stellen ist der Untergrund in stärkerem Maße steinig, und die dort gerade aufwachsenden Muschelbänke zeigen an, daß stärkere Umlagerungen nicht stattfinden. Hier breitet sich Chondrus crispus (L.) Stackh. neben den bereits genannten Algen aus, von denen Dumontia, Scytosiphon und Chaetomorpha diesen Standort deutlich bevorzugen. Die Steine sind vielfach bewachsen mit Ralfsia verrucosa Aresch., die oft von Isactis plana (Harv.) Thur. und Chantransia-Arten überzogen ist.

Ober den in Lebensgemeinschaft mit der Miesmuschel in einzelnen Büscheln auf dem Watt und in dichten Beständen auf den Muschelbänken im Königshafen überall anzutreffenden Fucus mytili Nienbg. hat NIENBURG (1927, 1932) berichtet. Er bildet nur selten Luftblasen aus und fruktifiziert unregelmäßig; die Konzeptakel enthalten nur Oogonien, die keine reifen Eier entlassen. Auf diesem Fucus trifft man vom Frühjahr bis zum Herbst stets Elachista fucicola (Vell.) Aresch. an, die ihrerseits häufig Ectochaete Wittrockii (Wille) Kylin trägt. Chaetomorpha linum (Müll.) Küts. ist eine typische Wattenmeeralge, die zu dichten, verworrenen Fadenknäueln zusammengedreht im Watt treibt und dort auf den Muschelbänken hängen bleibt. Nach Stürmen findet man sie oft in meterlangen Strängen im Spülsaum am Strande.

Der Bewuchs auf den Pfählen der Landungsbrücke weist keine Besonderheiten auf. Sie sind der Standort für Pylaiella litoralis (L.) Kjellm., Enteromorpha-Arten und grüne Fadenalgen. Porphyra umbilicalis (L.) Kü̧. tritt hier zuweilen in dichten Beständen auf.

Fucus vesiculosus $\mathrm{L}$. findet sich an den Wänden des Lister Hafens und an gemauerten Uferbefestigungen, außerdem auf einer Ablaufbahn, die auf die Reede führt. Auf dieser schwach geneigten Betonfläche wurden neben vielen der bereits genannten Arten auch Monostroma Grevillei (Thur.) Wittr., Phyllitis fascia (Müll.) Küt., Punctaria latifolia Grev. und Myriotrichia cla- 
vaeformis Harv., letgtere als Epiphyt auf Scytosiphon, festgestellt. Im obersten Litoral trifft man Capsosiphon fulvescens (C. Ag.) Setch. und Gard. zwischen Enteromorpha minima an.

Im sublitoralen Bereich tritt Bryopsis hypnoides Lamour. regelmäßig in den Frühjahrsmonaten an den Pfählen der Anlegebrücken im-Hafen auf. Nicht häufig findet sich Laminaria saccharina (L.) Lamour. zu beiden Seiten der Ablaufbahn.

Die folgende Aufstellung bringt eine Ergänzung zu den bisher im Text aufgeführten Arten. Die Liste ist damit nicht vollständig, einzelne Funde bedürfen noch einer eingehenderen Untersuchung auf ihre Artzugehörigkeit.

Gr ün algen:

Enteromorpha intestinalis (L.) Link An verschiedenen Stellen der Insel, besonders in Gräben im Marschland bei List.

Enteromorpha torta (Mert.) Reinb.

Besiedelt gern die Abbruchkanten an exponierten Stellen der Wattenmeerküste. Häufig in einer schlickigen Lagune.

Percursaria percursa (Ag.) Rosenv.

Mit anderen fädigen Grünalgen zusammengesponnen auf etwas schlickhaltigen Wattflächen. Teilt den Standort mit Enteromorpha torta.

Cladophora Hutchinsiae (Dillw.) Küty.

Cladophora flexuosa (Griff.) Harv.

Cladophora sericea (Huds.) Kütg. ?

Alle nicht häufig am Nordstrand.

Cladophora lanosa (Roth) Küts.

An alten Buhnenpfählen der Westküste.

Cladophora fracta (Fl. Dan.) Küts., f. marina Hauck. Regelmäßig in den Aquarienbecken.

Prasinocladus lubricus Kuck.

In großen Mengen in den Aquarien.

Braunalgen:

Mikrosyphar porphyrae Kuck.

Selten auf Porphyra umbilicalis.

Myrionema strangulans Grev.

Häufig auf Enteromorpha-Arten.

Chordaria flagelliformis (Müll.) Ag.

Nur einmal im Gebiet festgewachisen gefunden.

Rotalgen :

Erythrotrichia carnea (Dillw.) J. Ag.

Epiphytisch auf Rhodophyceen.

Goniotrichum elegans (Chauv.) Le Jol.

Wie vorige, aber viel seltener.

Polysiphonia violacea (Roth) Grev.

Nur zweimal auf ihrem Substrat festgewachsen und einmal treibend gefunden.

Lithothamnion spec.

Auf Steinen und lebenden Miesmuscheln sublitoral bei der Anlegebrücke am Ellenbogen. 
Ein Vergleich mit früheren Beobachtungen läßt erkennen, daß sich die Algenflora von List in den leţten Jahrzehnten ziemlich stark verändert hat. Bis 1934 besiedelte das große Seegras (Zostera marina L.) den Königshafen in ausgedehnten Beständen (vgl. Nienburg, 1927 und WoHLENBERg 1935). Blätter und Rhizome gaben manchen Algen Ansatzmöglichkeit. Die heute nahezu verschwundene Polysiphonia violacea war nach Kuckuck's Exkursions-Tagebüchern aus den Jahren 1896 und 1898 häufig im Königshafen an Seegras zu finden, NiEnburg (1927) gibt sie ebenfalls für die Seegraswiesen an, und Wohlenberg (1937) erwähnt ihr Vorkommen auf Chorda filum. Von Kuckuck wurden im Königshafen noch gefunden: Leathesia difformis (L.) Aresch., Castagnea virescens (Carm.) Thur., Dictyosiphon foeniculaceus (Huds.) Grev., Sphacelaria cirrhosa (Roth) Ag. var. aegagropila Griff. (auch von Reinbold, 1892, angegeben) und Rhodomela subfusca (Woodw.) Ag. Melobesia wurde von Magnus (1872), KuckucK und NiENBURg (1927) auf Zosterablättern gefunden.

Uber das Auftreten von Dictyota dichotoma berichtet HAGmeIer (1941). Die Alge kam auf der Ellenbogenbank seit der Einfuhr der ersten holländischen Saataustern im Jahre 1925 vor. 1932 trat sie in größerer Menge auf, so daß sie als Charakterart für die Austernbank bezeichnet wurde. Ein noch seltenerer und eigenartiger Gast des Wattenmeeres ist Codium tomentosum (Huds.) Stackh., von dem 1932 zwei kleine Pflänzchen auf lebenden Austern gefunden wurden.

Dieser Gruppe von Algen, die verschwunden sind oder die nur vorübergehend auftraten, steht die viel größere der Arten gegenüber, die früher nicht für Sylt angegeben wurden. Sollten sie damals gefehlt haben? Es ist - zumindest für einen Teil der Arten - recht unwahrscheinlich. Wenn auch durch Kunstbauten für einen Teil des jetzt vorhandenen Bestandes erst die Anwuchsmöglichkeit geschaffen wurde, so liegt doch die Annahme nahe, daß die sandigen Wattflächen im Süden der Ellenbogen-Halbinsel seit langer Zeit die aufgelockerte Algenvegetation tragen, wie wir sie auch heute antreffen. KuCKUCK ist auf seinen Exkursionen von List nur bis an den Priel gelangt, und auch ReINBold hat die Wattflächen nördlich des Priels offenbar nicht untersucht. Die heutige Algenvegetation dieses Gebiets enthält im wesentlichen die gleichen Arten, die von Reinbold (1892) und nach KuCKuck's ExkursionsTagebüchern bei Amrum und Föhr vorgefunden wurden.

Eine reichhaltige Ergänzung der lokalen Flora bietet der Algenbewuchs an treibenden Gegenständen und die nach anhaltenden Stürmen aus nördlicher Richtung of tmals in größeren Mengen am Strande angetriebenen Algen. Sie stammen - wenigstens zum Teil - wahrscheinlich von den Küsten Englahds und Schottlands. Rosenvinge' (1906) gab eine Zusammenstellung von 48 an der dänischen Westküste angetriebenen Algenarten, meist Epiphyten an Ascophyllum nodosum oder an den Bechern von Himanthalia lorea. $30 \mathrm{der}$ von ihm längs der Küste von Fanö bis Skagen gefundenen Arten wurden auch in Sylt angetrieben, während 18 Arten bei uns noch nicht festgestellt wurden. Darüber hinaus aber enthält unsere Sammlung weitere 28 einwandfrei bestimmbare Arten, darunter die im Herbst häufigen Ectocarpus velutinus und Elachista scutulata und als seltenere Vertreter Leathesia difformis und Corallina squamata. In sehr vielen Fällen erlaubt das wenige Material keine genaue Artbestimmung, besonders, wenn die Fortpflanzungsorgane fehlen. 
Zuweilen findet man größere Mengen von Laminaria hyperborea und L. saccharina zusammen mit Desmarestia aculeata und Delesseria sanguinea am Weststrand und an der Nordspitze der Insel, ohne daß gleichzeitig Ascophyllum und Himanthalia antreiben. Die völlig frischen Laminarien zeichnen sich durch ein hochgewölbtes Knäuel dünner, reichverzweigter Haftkrallen aus, während die Laminarien von Helgoland viel derbere und mehr flächig ausgebreitete Hafter haben. Aus ihrem Auftreten ohne die gut schwimmfähigen Fucaceen ist zu schließen, daß sie nicht von entfernten Küsten stammen; nach der Form ihrer Haftkrallen dürften sie wohl auf hartem Gestein gewachsen sein. Es wäre nicht ausgeschlossen, daß westlich der Insel Moränenmaterial des abgetragenen Geestkernes liegt, auf dem sie vorkommen könnten.

\section{Literatur}

Hagmeier, A., Die intensive Nutzung des nordfriesischen Wattenmeeres durch Austernund Muschelkulturen. Ztschr. f. Fischerei u. d. Hilfswissensch. 39, 1941.

Magnus, P., Die botanischen Ergebnisse der Nordseefahrt vom 21. Juli bis 9. Sept. 1872. II. Ber. Komm. z. Unters. deutsch. Meere, Berlin 1874.

Nienburg, W., Zur Okologie der Flora des Wattenmeeres. I. Teil. Der Königshafen bei List auf Sylt. Wiss. Meeresunters. Abt. Kiel 20, 1927.

Nienburg, W., Fucus Mytili spec. nov. Ber. D. Bot. Ges., 50 a, 1932.

Reinbold, Th., Beiträge zur Kenntnis der Algenvegetation des östlichen Theiles der Nordsee. Schriften des Naturwissenschaftlichen Vereins für Schleswig-Holstein, 9, 1892.

Rosenvinge, L. K., Om fremmede Alger ilanddrevne paa Jyllands Vestkyst. Botanisk Tidsskrift, 27, Kopenhagen, 1906.

- Distribution of the Rhodophyceae in the Danish Waters. Danske Vidensk. Selsk. Skrifter. Naturv. og Mathem. Afd., 9. Raekke, 6, 1935.

Wohlenberg, E., Beobachtungen über das Seegras, Zostera marina L., und seine Erkrankung im nordfriesischen Wattenmeer. Nordelbingen, Beitr. z. Heimatforschung in Schlesw.-Holstein, Hamburg und Lübeck. 11, 1935.

- Die Wattenmeerlebensgemeinschaften im Königshafen von Sylt. Helg. Wiss. Meeresunters. $1,1937$. 